\title{
PATTERN-GENERATOR-DRIVEN DEVELOPMENT IN SELF-ORGANIZING MODELS
}

\author{
James A. Bednar and Risto Miikkulainen \\ Department of Computer Sciences \\ The University of Texas at Austin \\ Austin, TX 78712 \\ jbednar, risto@cs.utexas.edu
}

\begin{abstract}
Self-organizing models develop realistic cortical structures when given approximations of the visual environment as input. Recently it has been proposed that internally generated input patterns, such as those found in the developing retina and in PGO waves during REM sleep, may have the same effect. Internal pattern generators would constitute an efficient way to specify, develop, and maintain functionally appropriate perceptual organization. They may help express complex structures from minimal genetic information, and retain this genetic structure within a highly plastic system. Simulations with the RF-LISSOM orientation map model indicate that such preorganization is possible, providing a computational framework for examining how genetic influences interact with visual experience.
\end{abstract}

\section{INTRODUCTION}

Many self-organizing computational models of cortical development have been proposed in recent years ${ }^{1,2}$. The most common type of such models shows that simple activitydependent learning processes can result in the development of realistic cortical structures. For instance, the RF-LISSOM model ${ }^{3-6}$ is trained using Hebbian learning with simulated visual inputs, and the initially undifferentiated neurons and connections in the model selforganize into orientation, ocular dominance, and size-selective columns with patchy lateral connections between them. Models of this type give computational support to the idea that the cortex organizes to represent and process regularities in the visual input ${ }^{7,8}$.

Despite a common visual environment, different species develop cortical architectures with a variety of functional properties ${ }^{9}$. Such idiosyncrasies are generally beneficial to each species. For instance, a predator such as a cat develops a preponderance of cortical motion detectors, while fruit-eating animals such as monkeys devote more neurons to the representation of form. Cortical areas within a single animal also develop similar specializations, even though some receive their primary input from a common source.

This article considers how such a variety of architectures could develop in a self-organizing system. In the RF-LISSOM approach, the large-scale structure of the network is geneti- 

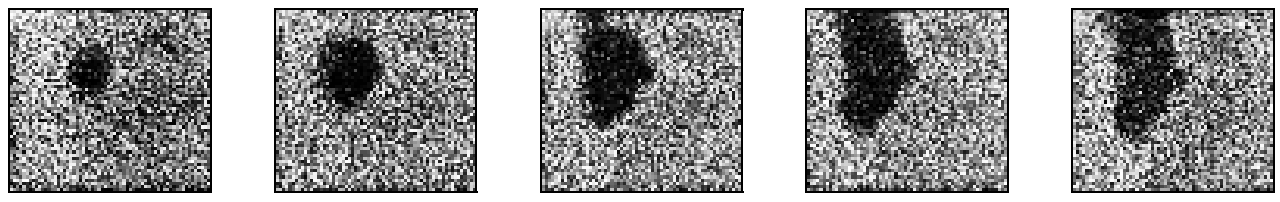

Figure 1. Spontaneous wave in the ferret retina. Each of the frames shows approximately $1 \mathrm{~mm}^{2}$ of ferret retina using calcium concentration imaging, a measure of the activity of the retinal cells. Dark areas indicate increased activity. From left to right, the frames form a 2 -second sequence showing the start and expansion of a spontaneous retinal wave. (Reprinted with permission from Feller et al. "Requirement for cholinergic synaptic transmission in the propagation of spontaneous retinal waves", Science, 272:1182-1187, 1996. Copyright 1996 American Association for the Advancement of Science.)

cally specified. Self-organization operates within this structure and develops local functional properties based on input activation. A variety of results can be obtained if one of the three basic components of the self-organizing system is varied: the initial configuration, the learning mechanism, or the training inputs.

The initial configuration may be an important source of variation. Highly-specific connection patterns have been found at birth in many cortical areas ${ }^{10}$. However, there is clearly not enough space available in the genome of a mammal (on the order of $10^{5}$ genes) to specify every connection in its nervous system (as many as $10^{15}$ connections) ${ }^{11}$. Thus specific genetic hardwiring cannot fully explain systematic inter-species differences ${ }^{12}$. Neither can differences in learning mechanisms, since these mechanisms appear to be highly similar among mammalian species and brain areas ${ }^{13}$.

Cortical training inputs, on the other hand, are quite likely to differ substantially between species and brain areas. For one thing, retinal cells of different species have different distributions of spatial and temporal response properties, which would cause different patterns of activity to be seen by higher areas. In addition, different species might have genetic predispositions to attend to certain features of the visual input, thus automatically selecting different training distributions.

Even more systematic differences in training inputs are possible, however, if training inputs are generated internally under genetic control ${ }^{12,14-18}$. Instead of precisely specifying cortical organization, the genome may simply encode a developmental process that is based on patterns presented to self-organizing mechanisms. Candidate patterns have been found in two regions that send input to the visual cortex: the developing retina and the brain stem. These two sources may serve different purposes in a self-organizing system: preorganization into genetically specified structures before birth, and maintenance of these structures in later life while simultaneously allowing a large degree of cortical plasticity. Simulations using the RF-LISSOM model demonstrate how the cortex could self-organize using retinal waves, and represent a step towards understanding genetic expression within a highly adaptive system.

\section{SPONTANEOUS RETINAL WAVES}

The best-documented source of generated patterns is the developing retina, where the patterns take the form of intermittent spatially-coherent activity waves across groups of ganglion cells ${ }^{19,20}$. Similar waves have also been documented in other early sensory areas, such as the auditory systems of birds ${ }^{21}$. A number of recent experiments strongly suggest that the pattern of retinal activity waves is responsible for the segregation of the LGN into eyespecific layers before birth ${ }^{12,18}$.

The spontaneous retinal waves may also represent the earliest activity seen by the de- 

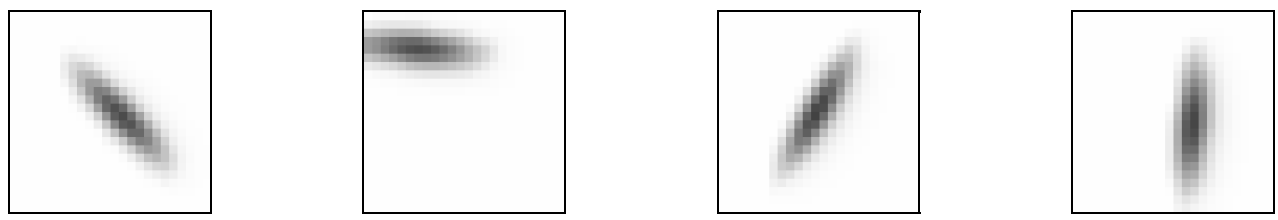

Figure 2. Sample RF-LISSOM training inputs plotted on the retina. Simple Gaussian patterns similar to retinal waves (figure 1) are used to train the model. Each presentation represents a single retinal wave, with position and orientation chosen at random. The temporal behavior of the waves is not currently modeled, only the approximate shape.

veloping visual cortex ${ }^{22}$. Visual input is known to have dramatic effects on the organization of the cortex ${ }^{23}$, a process which has also been demonstrated in self-organizing models ${ }^{3,24,25}$. One would expect that similar reorganization also occurs in response to the early, non-visual inputs. One purpose for the retinal waves could be to substitute for visual experience before eye-opening, thus minimizing the amount of time required to develop visual competence ${ }^{18}$. The precise shape and temporal behavior of the waves might also serve to guide development in a species-specific way by exploiting the same learning mechanisms which later incorporate environmental influences ${ }^{18}$.

\section{PGO WAVES IN REM SLEEP}

Once appropriate structures have been developed, internal pattern generation may help ensure that genetically-specified architectures remain, even though the system is continually adapting to the visual environment ${ }^{26}$. Presumably, such patterns would need to be presented when the system is not processing visual input, such as during sleep. Interestingly, the amount of rapid eye movement (REM) sleep is strongly correlated with the degree of neural plasticity across phylogeny and ontogeny ${ }^{17,26}$. Thus one function of REM sleep may be to present such training patterns for genetic expression and maintenance during neural adaptation ${ }^{17,26}$.

During and just before REM sleep, internally generated phasic waves called pontogeniculo-occipital (PGO) waves can be measured in the LGN, V1, and many other cortical areas ${ }^{26,27}$. The waves originate in the pons of the brain stem and travel via direct pathways to the LGN and visual cortex ${ }^{27}$, and appear to be relayed to many other areas of the cortex ${ }^{26}$. PGO waves are strongly correlated with eye movements as well as with vivid visual imagery in dreams, suggesting that they activate the visual cortex as if they were visual inputs ${ }^{16}$.

Furthermore, blocking these waves has been shown to heighten the effect of abnormal visual experience during development ${ }^{16}$. When the visual input to one eye of a kitten is blocked for a short time during a critical period, the cortical area devoted to signals from the other eye increases ${ }^{10}$. This effect is even stronger if REM sleep is also interrupted ${ }^{16}$, suggesting that PGO waves or other aspects of REM sleep ordinarily limit or counteract the effects of visual experience. As discussed below, one interpretation of these results is that PGO waves are part of a system for establishing and reinforcing genetically-specified architectures ${ }^{26}$.

\section{DEVELOPMENTAL EXPERIMENTS WITH RF-LISSOM}

Several preliminary experiments with internally-generated patterns have been conducted using the RF-LISSOM self-organizing model. When trained on randomly oriented Gaussian inputs similar to the spontaneous retinal waves (see figure 2), the model develops a realistic 
columnar orientation map (figure 3$)^{3,4}$. Thus the retinal patterns may explain how kittens have a crude version of such a map even when raised entirely in the dark ${ }^{10}$.

In animals, subsequent visual experience sharpens the map and greatly increases the number of orientation-selective cells ${ }^{10}$. This suggests that the visual environment has a larger percentage of strongly oriented inputs than are present in the retinal waves. Similar sharpening is observed in the RF-LISSOM model if it is first trained on less oriented inputs, then on more strongly oriented inputs ${ }^{6}$. Simulations with multiple simultaneous Gaussian inputs also suggest that early self-organization is improved if the Gaussians do not overlap ${ }^{6}$. This feature has subsequently been found in the retinal waves, which (unlike visual patterns) tend to occur singly in well-defined spatial domains ${ }^{20}$. Thus the internally-generated patterns and visual inputs together can provide a better basis for self-organization than either alone.

\section{DISCUSSION AND FUTURE WORK}

The simulations show that internally-generated patterns can be used successfully for self-organization. The pattern-generation approach represents a middle ground between full genetic specification of neural connections, at one extreme, and purely sensory-driven selforganization, at the other. Full genetic specification of structures as complex as the cortex would require an enormous number of genes, while specifying simple input patterns and learning mechanisms would take relatively few. For instance, the complicated structures seen in the self-organized model cortex (figure $3 b$ ) were generated using very simple patterns (figure 2). Furthermore, specification via pattern generation would be robust to random differences in the details of the external environment and the internal arrangement of cells and connections, unlike a literal encoding of specific connections.

From an evolutionary perspective, a literal encoding would require an implausibly large number of coordinated genetic changes before a viable yet functionally distinct architecture could evolve. Pattern generation, on the other hand, would allow species to differentiate via relatively small changes in the genome (i.e., only those portions encoding the input patterns). Jouvet ${ }^{26}$ has proposed that PGO waves are part of such a system for expression of individual and species-specific characteristics.

At the opposite extreme, a pure learning system would eventually overwrite any genetically encoded starting point. This would be undesirable since the information that is available to the organism directly from the environment is quite sparse compared to that from the millions of years contributing to the formation of the genome. Thus it would be better to retain some genetic guidance, regardless of how much visual experience is obtained - there will always be situations that an organism has not yet encountered. The genetic influences may act as a constraint upon the amount of learning that can occur, preventing the organism from becoming too specifically adapted to its particular circumstances at the expense of generality.

Thus the pattern-generation approach represents an efficient way to combine learning with genetic expression to develop a complex system. Taking into account the fact that a vertebrate genome is only somewhat larger than those of much simpler organisms ${ }^{9}$, one can even speculate that the combination of learning and pattern-generator-driven development was the key step that enabled the evolution of the complex nervous systems of higher vertebrates. At some point, evolution may have discovered how to trick learning into being a general mechanism for genetic and environmentally-controlled development.

Future RF-LISSOM simulations using patterns approximating PGO waves and other internally-generated waves should help clarify their effects upon cortical organization. In particular, different wave shapes and temporal characteristics could explain differences in functional properties among species. Through RF-LISSOM simulations, it is possible to determine what features in the input are crucial for self-organization. By comparing those 


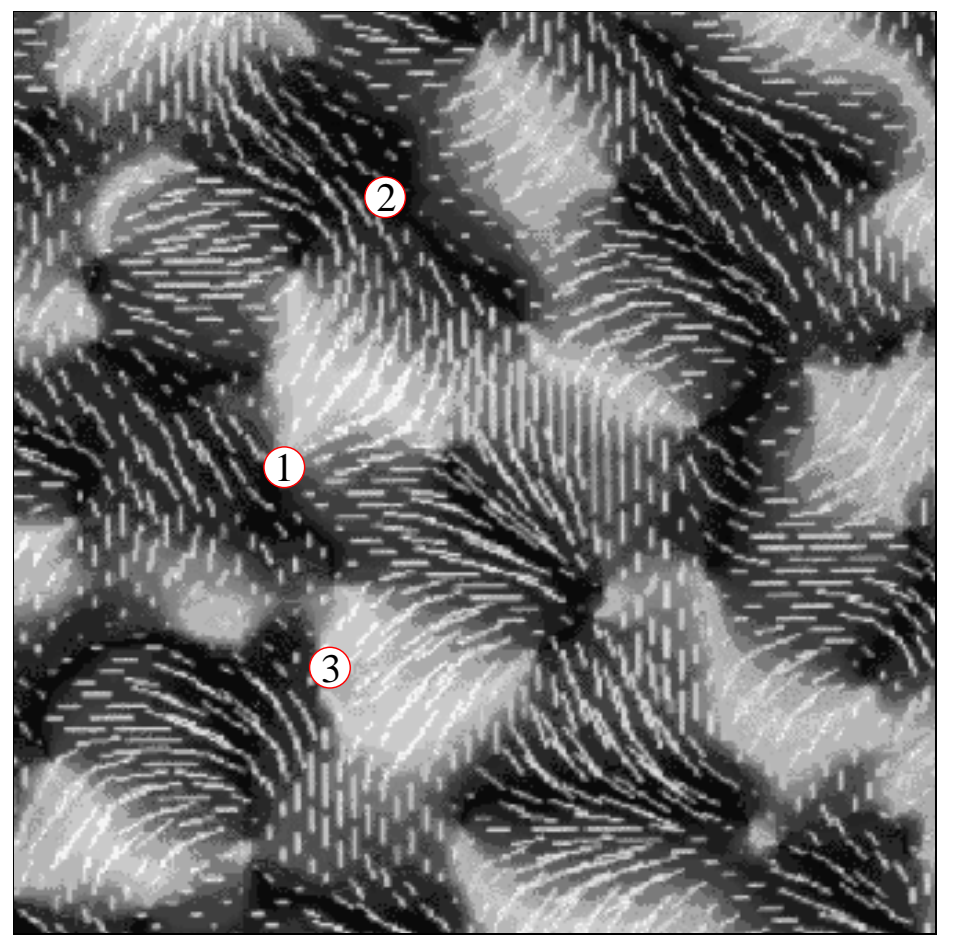

(a) Macaque orientation map

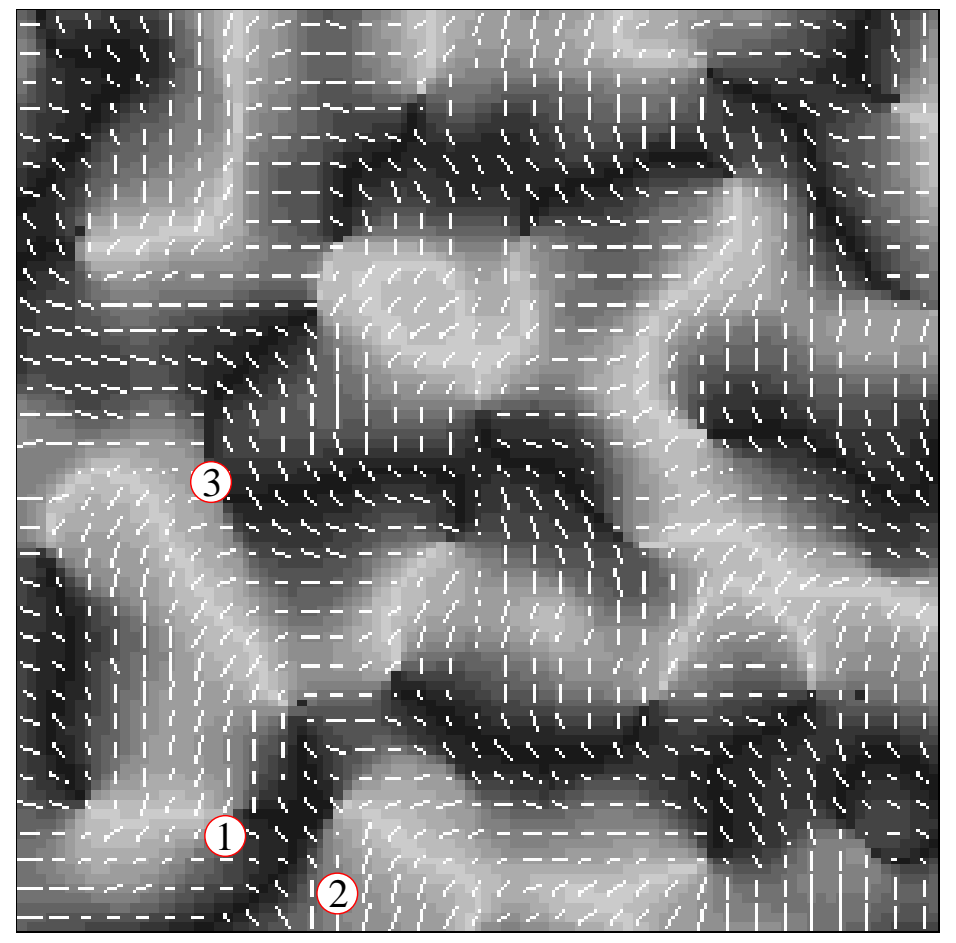

(b) RF-LISSOM orientation map 
Figure 3. Orientation Maps. (a) Orientation preferences of neurons in a $2.7 \times 2.7 \mathrm{~mm}$ area of the primary visual cortex of a macaque monkey. (Adapted with permission from figure 10A, Blasdel, "Orientation Selectivity, Preference, and...", The Journal of Neuroscience 12 (8): 3150, 1992.) (b) Orientation preferences of neurons in a $96 \times 96$ unit area of an RF-LISSOM map self-organized using inputs like those in figure $2^{4}$. In both figures, the white lines indicate the preferred orientation of the neuron, and the length of each line indicates the degree of orientation specificity of that neuron. The shading varies from black ( $45^{\circ}$ to the left of a vertical line pointing up) to light gray ( $45^{\circ}$ to the right). Both maps share important features (marked with numbered circles) such as (1) pinwheel centers, around which orientation preference changes through $180^{\circ}$, (2) linear zones, where orientation preference changes almost linearly, and (3) fractures, where there is a discontinuous change of orientation preference. The similarity of the model and experimental maps was also demonstrated using Fourier transforms, autocorrelation functions, and correlation angle histograms. A general discussion of these methods ${ }^{1}$, the relevant measurements for RF-LISSOM ${ }^{6}$, and an animation of the self-organizing process $^{3}$ are available.

features with ones known to be present in the visual environment or in internally generated patterns, more detailed hypotheses about the genetic and environmental basis of cortical structures can be formulated. Eventually, such simulations should help explain how and why the brain develops as it does in different animals.

\section{CONCLUSION}

We propose that internally-generated patterns represent a general mechanism that allows an organism to specify, develop, and maintain functional structures. RF-LISSOM simulations have shown how a crude initial orientation map could develop from non-visual inputs. Future work will examine how species differentiate, and how these genetic factors are maintained in the adult. Such experiments with self-organizing models should greatly improve our understanding of the balance between environmental and genetic determinants of individuality.

\section{ACKNOWLEDGMENTS}

This research was supported in part by the National Science Foundation under grant \#IRI-9309273. Computer time for the simulations was provided by the Pittsburgh Supercomputing Center under grant \#IRI940004P.

\section{REFERENCES}

1. E. Erwin, K. Obermayer, and K. Schulten, Models of orientation and ocular dominance columns in the visual cortex: A critical comparison, Neural Computation, 7(3):425-468 (1995).

2. N. V. Swindale, The development of topography in the visual cortex: A review of models, Network, 7:161247 (1996).

3. J. Sirosh, R. Miikkulainen, and J. A. Bednar, Self-organization of orientation maps, lateral connections, and dynamic receptive fields in the primary visual cortex, in: Lateral Interactions in the Cortex: Structure and Function, J. Sirosh, R. Miikkulainen, and Y. Choe, eds., The UTCS Neural Networks Research Group, Austin, TX (1996). Electronic book, ISBN 0-9647060-0-8, http://www.cs.utexas.edu/users/nn/webpubs/htmlbook96.

4. R. Miikkulainen, J. A. Bednar, Y. Choe, and J. Sirosh, Self-organization, plasticity, and low-level visual phenomena in a laterally connected map model of the primary visual cortex, in: Perceptual Learning, R. L. Goldstone, P. G. Schyns, and D. L. Medin, eds., volume 36 of Psychology of Learning and Motivation, 257-308, Academic Press, San Diego, CA (1997). 
5. J. Sirosh and R. Miikkulainen, Cooperative self-organization of afferent and lateral connections in cortical maps, Biological Cybernetics, 71:66-78 (1994).

6. J. Sirosh, A Self-Organizing Neural Network Model of the Primary Visual Cortex, Ph.D. thesis, Department of Computer Sciences, The University of Texas at Austin, Austin, TX (1995). Technical Report AI95-237.

7. H. B. Barlow, The twelfth Bartlett memorial lecture: The role of single neurons in the psychology of perception, Quarterly Journal of Experimental Psychology, 37A:121-145 (1985).

8. D. J. Field, What is the goal of sensory coding?, Neural Computation, 6:559-601 (1994).

9. D. Purves. Body and brain: A trophic theory of neural connections, Harvard University Press, Cambridge, MA (1988).

10. C. Blakemore and R. C. van Sluyters, Innate and environmental factors in the development of the kitten's visual cortex, Journal of Physiology (London), 248:663-716 (1975).

11. E. R. Kandel, J. H. Schwartz, and T. M. Jessell. Principles of Neural Science, third edition, Elsevier, New York (1991).

12. C. J. Shatz, Emergence of order in visual system development, Proceedings of the National Academy of Sciences, USA, 93:602-608 (1996).

13. A. Kirkwood and M. F. Bear, Hebbian synapses in visual cortex, Journal of Neuroscience, 14(4):16341645 (1994).

14. M. Constantine-Paton, H. T. Cline, and E. Debski, Patterned activity, synaptic convergence, and the NMDA receptor in developing visual pathways, Annual Review of Neuroscience, 13:129-154 (1990).

15. L. Maffei and L. Galli-Resta, Correlation in the discharges of neighboring rat retinal ganglion cells during prenatal life, Proceedings of the National Academy of Sciences, USA, 87:2861-2864 (1990).

16. G. A. Marks, J. P. Shaffery, A. Oksenberg, S. G. Speciale, and H. P. Roffwarg, A functional role for REM sleep in brain maturation, Behavioural Brain Research, 69:1-11 (1995).

17. H. P. Roffwarg, J. N. Muzio, and W. C. Dement, Ontogenetic development of the human sleep-dream cycle, Science, 152:604-619 (1966).

18. C. J. Shatz, Impulse activity and the patterning of connections during CNS development, Neuron, 5:745756 (1990).

19. M. Meister, R. O. L. Wong, D. A. Baylor, and C. J. Shatz, Synchronous bursts of action-potentials in the ganglion cells of the developing mammalian retina, Science, 252:939-943 (1991).

20. M. B. Feller, D. P. Wellis, D. Stellwagen, F. S. Werblin, and C. J. Shatz, Requirement for cholinergic synaptic transmission in the propagation of spontaneous retinal waves, Science, 272:1182-1187 (1996)

21. W. R. Lippe, Rhythmic spontaneous activity in the developing avian auditory system, Journal of Neuroscience, 14(3):1486-1495 (1994).

22. R. Mooney, A. A. Penn, R. Gallego, and C. J. Shatz, Thalamic relay of spontaneous retinal activity prior to vision, Neuron, 17:863-874 (1996).

23. J. A. Movshon and R. C. van Sluyters, Visual neural development, Annual Review of Psychology, 32:477522 (1981).

24. G. Goodhill, Topography and ocular dominance: A model exploring positive correlations, Biological Cybernetics, 69:109-118 (1993).

25. C. von der Malsburg, Self-organization of orientation-sensitive cells in the striate cortex, Kybernetik, 15:85-100 (1973).

26. M. Jouvet, Paradoxical sleep and the nature-nurture controversy, in: Adaptive Capabilities of the Nervous System, P. S. McConnell, G. J. Boer, H. J. Romijn, N. E. van de Poll, and M. A. Corner, eds., volume 53 of Progress in Brain Research, 331-346, Elsevier, New York (1980).

27. M. Steriade, D. Paré, D. Bouhassira, M. Deschênes, and G. Oakson, Phasic activation of lateral geniculate and perigeniculate thalamic neurons during sleep with ponto-geniculo-occipital waves, Journal of Neuroscience, 9(7):2215-2229 (1989). 